\title{
Angiospermic flora of Borail Wildlife Sanctuary (BWS) in Assam, India : First report
}

\author{
A. Kar ${ }^{1}$, D. Boruah, N. K. Goswami and D. Saharia \\ The Energy and Resources Institute (TERI), North Eastern Regional Centre, Chachal, VIP Road, \\ Hengrabari, Guwahati-781 036, Assam, India \\ ${ }^{1}$ Corresponding author: E-mail: ashishvision20@gmail.com \\ [Received 29.11.2018; Revised 18.12.2018; Accepted 23.12.2018; Published 31.12.2018]
}

\begin{abstract}
The present investigation deals with the composition of angiosperm plants in the Borail Wildlife Sanctuary, Assam. A total of 281 angiosperm plant species under 199 genera and 64 families were recorded from the sanctuary during the survey period. Angiosperm included herbs (84), trees (83), shrubs (45), climber (38), creeper (01), lianas (6), palm (3), grass (10), sedge (6) and bamboo (5) species.
\end{abstract}

Key words: Angiosperm flora, Borail Wildlife Sanctuary, Assam

\section{INTRODUCTION}

Protected areas help to reduce forest fragmentation and degradation through protection laws and best management practices for biodiversity conservation. Wildlife sanctuary is endowed with ecological, floral, faunal and geomorphologic significance (Shukla 2016). Protected areas helps in recharging ground water, offer scope for pollination of commercially valuable crops, act as carbon sink, helps in soil stabilization etc. (Noss 1992). In Wildlife sanctuary, animals are brought to life and protected for the rest of their life until their natural death (Seikh 2018).

Assam has an geographical area of 78,433 sq. km, lying in between $24^{\circ} 44^{\prime} \mathrm{N}$ to $27^{\circ} 45^{\prime} \mathrm{N}$ latitude and $89^{\circ} 41^{\prime}$ and $96^{\circ} 02^{\prime} \mathrm{E}$ longitudes. The state is surrounded by hills and mountains on the north, east and the south side. To the west, it merges with the West Bengal and Bangladesh plains. The state has the Brahmaputra valley in the northern part bordering to Arunachal Pradesh and the Barak valley in the southern part bordering to Mizoram, Tripura and Meghalaya. The state is enriching with 3832 (dicotyledons 2752 and monocotyledon 1080) species of angiosperm plants comprising of 1370 (dicotyledons 1012 and monocotyledon 368) genera distributed in 229 (dicotyledons 189 and monocotyledon 40) families (Chowdhury 2005).

Barail Wildlife Sanctuary (BWS) is situated in the northern part of Cachar district of Assam and lies along the foothills of the North Cachar and Barail hills (Plate:1). The sanctuary is located between latitude $24^{\circ} 58^{\prime}-25^{\circ} 50^{\prime} \mathrm{N}$ and longitude $92^{\circ} 50^{\prime}-92^{\circ} 52^{\prime} \mathrm{E}$. The total area covered by this wildlife sanctuary is $326.24 \mathrm{sq} \mathrm{km}$. Barail Wildlife Sanctuary is a combination Barail Reserve Forest and North Cachar Reserve Forest, which were together upgraded to Barail Wildlife Sanctuary in June 2004. The BWS is under the administrative control of the Southern Assam Forest Circle, Silchar, and consists of Barail Reserve Forest, which is part of the Cachar Forest Division (East Block) and North Cachar Reserve Forest, part of the Karimgunj Forest Division (West Block). The BWS is governed under two forest range 
offices namely, Udharbond and Kalain range offices (Hussain 2015; Bora \& Bhattacharyya 2017; Bora et al. 2017).

The climate is humid tropical to sub-tropical; annual rainfall varies from $200 \mathrm{~cm} /$ year to $600 \mathrm{~cm} /$ year and the average humidity is $72-90 \%$. Temperatures range minimum $8^{\circ} \mathrm{C}$ and maximum temperatures $38^{\circ} \mathrm{C}$. Major rivers draining the sanctuary are the Jatinga (Photo1), Daloo (Photo-24), Kayong, Gumra, and Boleswar. The sanctuary is characterized by undulating hills having altitudinal range of less than $30 \mathrm{~m}$ to more than $1867 \mathrm{~m}$. Geologically, the soils are sandy stony to clayey. The area is one of the richest treasure houses of flora as well as fauna due to its unique geographical position, diverse landscapes, wide range of physiographic conditions and high precipitation. As per classification of Champion and Seth (1968), the sanctuary has 2 broad groups of forests, tropical wet evergreen (Photo - 27) and tropical semi-evergreen forests. Tree, shrub and lianas species forming a thick vegetation of the sanctuary, Forest floor is enriched by many herbaceous species. The main secondary landscape elements are cultivated flatland, extensive bamboo brakes (Photo-26), tree plantations (Teak and Sal), secondary and disturbed forest (betel-vine plantation), and village gardens including Areca nut plantations (Barbhuiya \& Singh 2012; Hussain 2015; Bora \& Bhattacharyya 2017; Bora et al. 2017).

Several publications came out on assessment of plant diversity in protected areas of Assam. Some of them are Sarkar \& Devi (2015) on Assessment of plant diversity in Hollongapar Gibbon Wildlife Sanctuary; Jain \& Hajra (1975) on the plant diversity of Manas Wildlife Sanctuary; Dutta et al. (1974) on forest flora of North Cachar Hills and Borail Range; Buragohain \& Swargiari (2016) on Diversity and conservation of Ficus Linnaeus (Moraceae) in Chakrashila Wildlife Sanctuary; Baruah \& Baruah (2000) on hydrophytes of Kaziranga National Park; Baruah \& Baruah (2007) on vegetation characteristics of grassland of Kaziranga National Park; Baruah et al. (2003) on biodiversity status in Manas Biosphere reserve; Bharali \& Borua (2003) on diversity of orchid flora of Dibru-Saikhowa National Park and Biosphere Reserve; Gogoi et al. (2009) on orchid flora of Joypur Reserve Forest of Dibrugarh district; Gogoi et al. (2009) on orchid flora of Dibru-Saikhowa National Park and Biosphere Reserve; Gogoi (2005) on Dendrobium genus of Dibru-Saikhowa National park and Biosphere Reserve; Dey et al. (2007) on Orchid diversity in Manas National Park, Assam; Bujarbarua \& Sarma (2006) on the diversity of family Poaceae in Gibbon Wildlife Sanctuary, Assam; Konwar et al. (2009) on abundance of food plant species and food habits of Rhinoceros unicornis L. in Pobitora Wildlife Sanctuary; Talukdar \& Deori (2017) on Floristic Diversity of Laokhowa Wildlife Sanctuary; Nath (2012) on Aquatic macrophytes of Laokhowa Wildlife Sanctuary; Deori \& Talukdar (2015) Floristic Diversity of Barnadi Wildlife Sanctuary; Kar et al. (2015) on vascular plant diversity in Amchang Wildlife Sanctuary

As far as plant diversity study of the Borail Wildlife Sanctuary is concerned, only few report on angiosperm plants (Dutta et al. 1974); on observations of trees and lianas of Borail Wildlife Sanctuary (Bora \& Bhattacharyya 2017); on Grasses and bamboos of Barail Wildlife Sanctuary (Bora et al. 2017) is available. There is no report of herbaceous plant is available, thus this work is attempt to provide information all the herb, shrub, tree, lianas and epiphytes, cane $\&$ bamboo. Therefore, present investigations were carried out with the objective- study of angiosperm plant diversity of Borail wildlife Sanctuary.

\section{MATERIALS AND METHODS}

Detailed surveys of the angiosperm plants of Borail Wildlife Sanctuary were conducted from April 2018 to December 2018 covering wild species. Surveys were conducted from nine sites of the sanctuary mostly from East block viz., Madhura, Indranagar, Khasia Punji, 

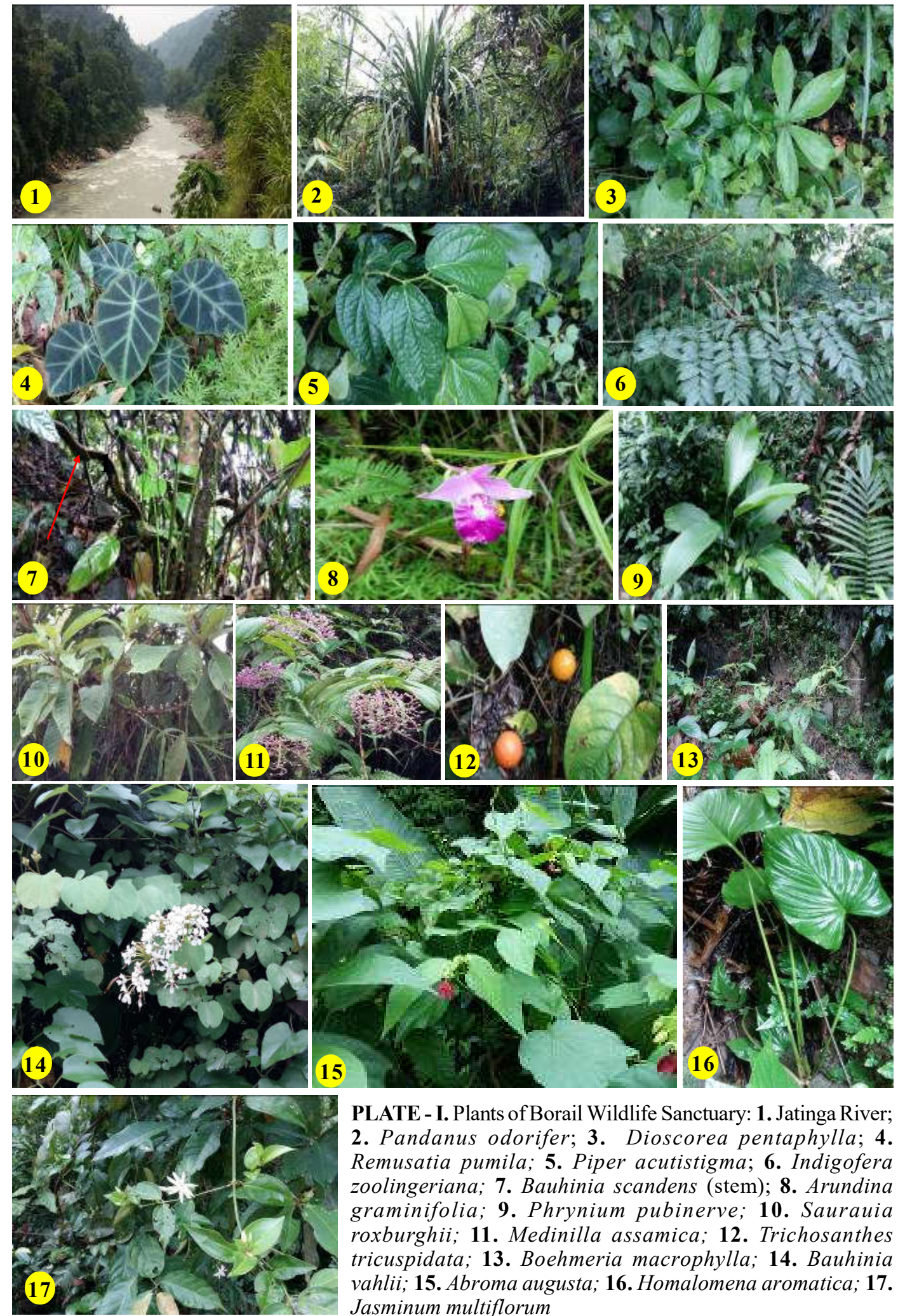

PLATE - I. Plants of Borail Wildlife Sanctuary: 1. Jatinga River; 2. Pandanus odorifer; 3. Dioscorea pentaphylla; 4. Remusatia pumila; 5. Piper acutistigma; 6. Indigofera zoolingeriana; 7. Bauhinia scandens (stem); 8. Arundina graminifolia; 9. Phrynium pubinerve; 10. Saurauia roxburghii; 11. Medinilla assamica; 12. Trichosanthes tricuspidata; 13. Boehmeria macrophylla; 14. Bauhinia vahlii; 15. Abroma augusta; 16. Homalomena aromatica; 17. Jasminum multiflorum 


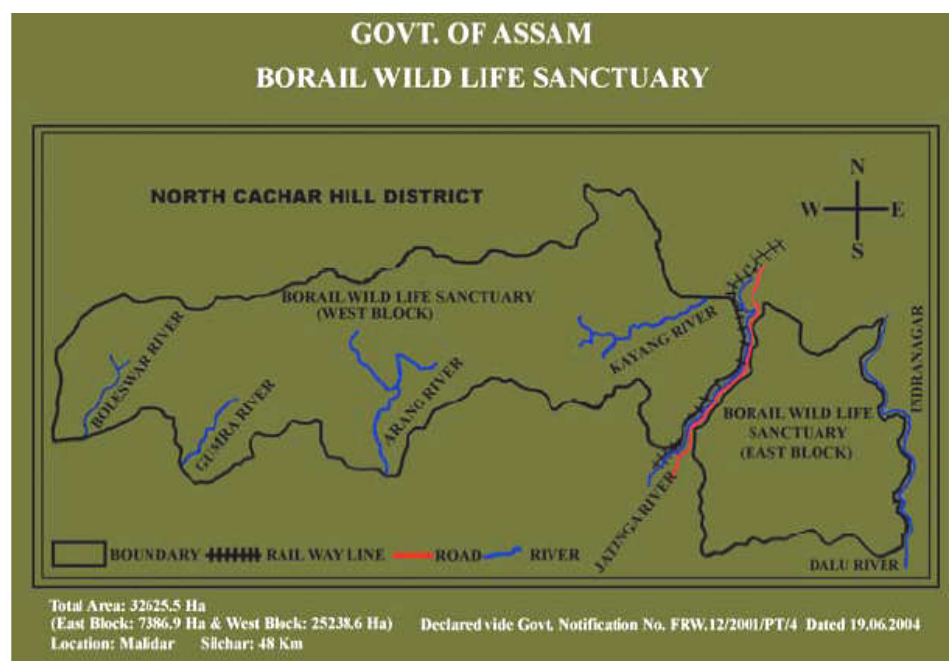

Figure 1. Map (not to scale) of the study area

Kapocherra, Balacherra, Marwa cherra, Durbin tilla, and dense forest areas of the East Block and in West block Damcherra and Bandarkhal area. External mor-phological characteristics including hairs, stipules and floral parts, of collected specimens was observed using Foldscope microscope. Characteristics of each species were observed and noted. The collected specimens were processed into mounted herbarium specimen following standard herbarium techniques (Jain \& Rao 1977). Identification of the specimens was done by comparing the field descriptions and observations with the descriptions available in authentic literature (Kanjilal et al. 1934 - 1940; Chowdhury, 2005) and confirmed with ASSAM Herbarium, Shillong. Herbarium specimens were deposited at the TERI herbarium as a voucher specimen for future reference. The International Plant Name Index (IPNI 2012), The Plant List (2013) and Tropicos (2017) were consulted for current nomenclature of all taxa. The Angiosperm Phylogeny Group III Classifica-tion (APG III 2009) was followed for the classification of families. The families were arranged in alphabetical order.

\section{RESULTS AND DISCUSSION}

A total number of 281 species from 199 genera representing 64 families (Appendix I) were recorded from the study area. Angiosperm comprises of 64 families (dicots 50 and monocots 14), 199 genera (dicots 160 and monocots 39) and 281 species (dicots 224 and monocots 57). Out of the total recorded 281 angiosperm species the composition were herbs (84), trees (83), shrubs (45), climber (38), creeper (01), lianas (6), palm (3), grass (10), sedge (6) and bamboo (5) species.

Among dicotyledons, Asteraceae is the most dominant family in the study area in respect to number of species with (19 species), followed by Fabaceae with (18 species), Lamiaceae, Euphorbiaceae, Moraceae and Mimosaceae each with (11 species), Rubiaceae and Caesalpiniaceae each with (10 species) etc. In Monocot Poaceae is the dominant family with (15 species) followed by Araceae with ( 7 species), Cyperaceae and Dioscoreaceae each with (6 species), Zingiberaceae and Orchidaceae each with (4 species), Arecaceae and Smilacaceae each with (3 species). Among the dicotyledons Ficus is the dominant genera representing with 9 species followed by Clerodendrum and Albizia each with 6 species, Desmodium and Ipomoea each with 5 species. Among the monocotyledons most 

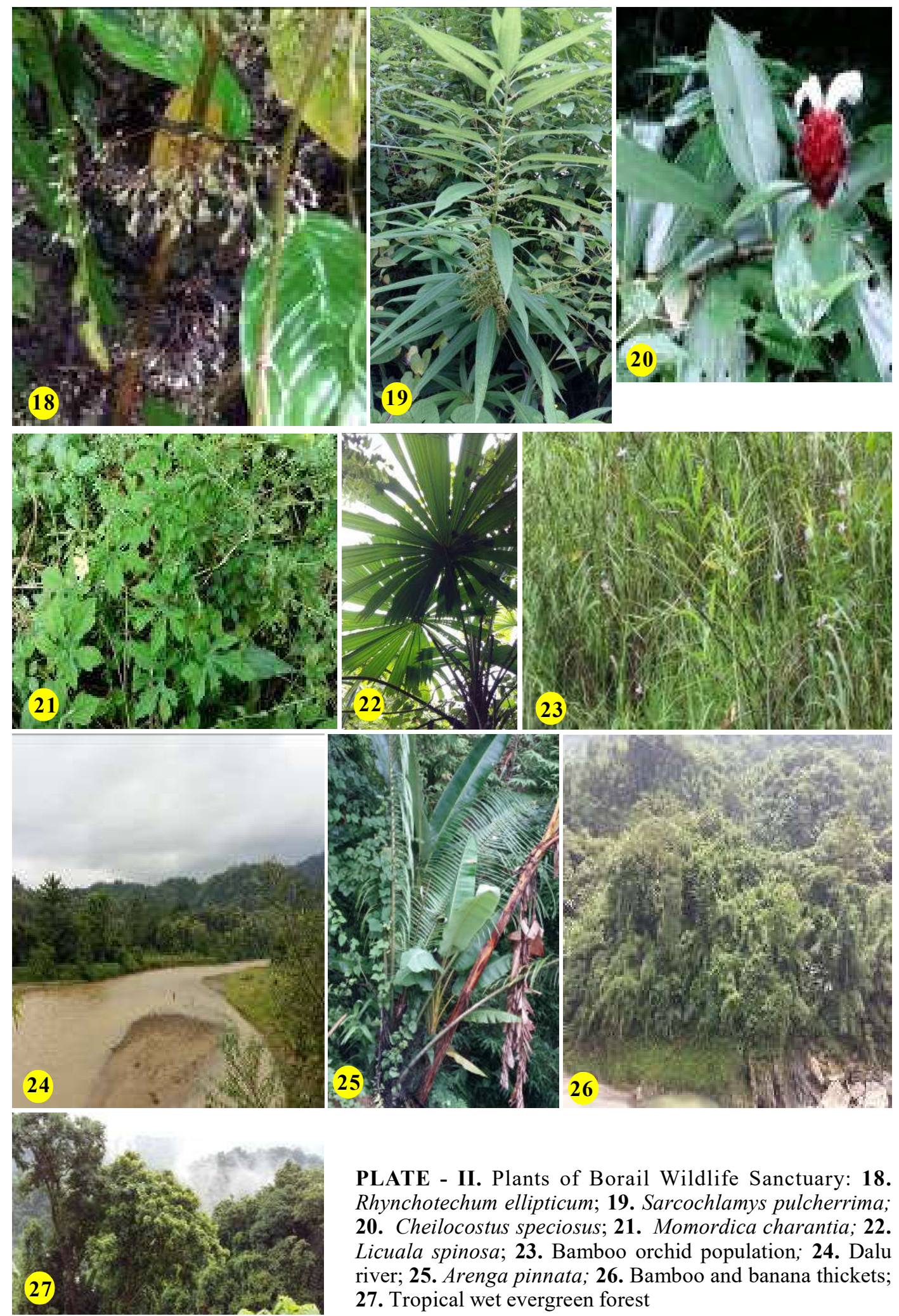

PLATE - II. Plants of Borail Wildlife Sanctuary: 18. Rhynchotechum ellipticum; 19. Sarcochlamys pulcherrima; 20. Cheilocostus speciosus; 21. Momordica charantia; 22. Licuala spinosa; 23. Bamboo orchid population; 24. Dalu river; 25. Arenga pinnata; 26. Bamboo and banana thickets; 27. Tropical wet evergreen forest 
Dioscorea is the most dominant genera representing with 6 species followed by Smilax, Bambusa, Cyperus and Globba each with 3 species.

\section{CONCLUSION}

It is interesting to note that Bauhinia scandens and Heterophragma adenophyllum each with only 2-3 plant recorded from the whole study area. Among tree, Callicarpa tomentosa, Tectona grandis, Sterculia villosa and among shrub, Clerodendrum infortunatum, Senna tora, Cayratia trifolia, Leea indica, Rhynchotechum ellipticum etc. Common herb species are Musa balbisiana, Stellaria media, Cynodon dactylon, Persicaria strigosa and among climber Thunbergia grandiflora, Ipomoea cheirophylla, Argyreia nervosa, Ipomoea purpurea etc. are very common in the study area.

It was observed that, there is severe pressure on the sanctuary due to human activities which is an alarming cause for decline in species diversity. Main problem of the sanctuary is deforestation due to construction of East-West Corridor which is passing through East block of the sanctuary. There is also the problem of stone quarrying, earth cutting which are highly detrimental to the flora and fauna of the sanctuary. Monoculture activities like Areca nut plantation, Tea plantation, and Teak plantation in fringe areas are also threat for the sanctuary. Thus, a multidimensional approach is required with regard to development of a conservation management plan for the safety of the sanctuary. Further research is required on ecology and population dynamics of the species of the sanctuary.

\section{Acknowledgements}

Authors are thankful to Office of the Chief Conservator of Forests, Southern Assam Circle, Assam for granting permission to carry out the study in Borail Wildlife Sanctuary. The first author (A. Kar) acknowledges the Department of Biotechnology (DBT), Govt. of India, New Delhi for financial support to undertake this study under the DBT-Foldscope programme.

\section{LITERATURE CITED}

APG III. 2009. An update of the Angiosperm Phylogeny Group clas-sification for the orders and families of flowering plants: APG III. Botanical Journal of the Linnean Society 161: 105-121. https://doi.org/10.1111/j.10958339.2009.00996.x

Barbhuiya, H.A. \& Singh, S.K. 2012. Liverwort and Hornwort of Borail Wild Life Sanctuary, Assam, India. Archive for Bryol. 134: 1 - 12.

Baruah, P.P. \& Baruah, C.K. 2000.Study of the hydrophytes flora of Kaziranga National Park, Assam, India. Ann. For. 8 (2): 170 - 178.

Baruah, P.P. \& Baruah, C.K. 2007.Vegetational characteristics of grassland of Kaziranga National Park, Assam, India. In: P.C. Trivedy (ed.), Global Biodiversity: status and conservation. Pointer Publication, Joipur. Pp. $210-219$.

Baruah, C.K.; Sharma G.C.; Bezbarua, P. \& Phukan, U. 2003. Biodiversity status in Manas Biosphere reserve. Report submitted to Min. of Enviroment \& Fores, Govt. of India, Pp. $1-40$.

Bharali, P. \& Borua, P.K. 2003. Diversity of orchid flora of Dibru-Saikhowa National Park and Biosphere Reserve, Assam. Book on 'Biodiversity of Eastern Himalayan Protected Areas' (edit), Dept. of Botany, Handique Girls’ College, Guwahati. Pp. $47-57$. 
Bora, A. \& Bhattacharyya, D. 2017. Phytodiversity of Barail Wildlife Sanctuary, Assam, India: field based observations- I. Trees and lianas. Check List 13 (6): 1037 1053.

Bora, A.; Devi, M. \& Bhattacharyya, D. 2017. Grasses and bamboos of Barail Wildlife Sanctuary in Assam, India. Pleione 11(2): 440 - 454.

Buragohain, R. \& Swargiari, B.N. 2016. Diversity and conservation of Ficus Linnaeus (Moraceae) in Chakrashila Wildlife Sanctuary, Kokrajhar District of Assam, India. Pleione 10(2): $302-309$.

Bujarbarua, P. \& Sarma, S.K. 2006. A note on the diversity of family Poaceae in Gibbon Wildlife Sanctuary, Assam, India. J. Econ. Taxon. Bot. 30(1): 1 - 5.

Champion, H.G. \& Seth, S.K. 1968. A revised survey of forest types of India, Govt. of India Press, Delhi.

Chowdhury, S. 2005. Assam's Flora (Present status of vascular plants), Published by Assam Science Technology and Environment Council, Guwahati

Deori, C. \& Talukdar, S.R. 2015. Floristic Diversity of Barnadi Wildlife Sanctuary, Assam. Nelumbo 57(2): $19-28$.

Dey, S.; Lakhar, B.P.; Das, J.P.; Nath, N.K. \& Brahma, N. 2007. Orchid diversity in Manas National Park, Assam. J. Orchid Soc. India 21(1-2): 65 - 68.

Dutta, A.K.; Dutta, T.K. \& Gupta, K.K. 1974. A tentative accounting of the forest flora of North Cachar Hills and Borail Range. Indian For. 100: 60 - 76.

Gogoi, K.; Borah, R.L. \& Sharma, G.C. 2009.Orchid flora of Joypur Reserve Forest of Dibrugarh district of Assam, India. Pleione 3(2): 135 - 147.

Gogoi, K.; Borah, R.L. \& Sharma, G.C. 2010. Orchid flora of Dibru-Saikhowa National Park and Biosphere Reserve, Assam, India. Pleione, 4 (1): 124 - 134.

Gogoi, K. 2005. The genus Dendrobium in Dibru-Saikhowa National park and Biosphere Reserve. J. Orchid Soc. India 19(1-2): 17 - 25.

Hussain, M.M. 2015. Technical Report: An Inventory Survey for the Forested IBAs in Barak Valley, Assam, (Supported by IBCN Small Grants 2014). Centre for Biodiversity and Natural Resource Conservation Department of Ecology \& Environmental Science Assam University, Silchar, Assam

IPNI 2012. The International Plant Names Index. http://www.ipni.org. [Accessed on: 0911-2018]

Jain, S.K. \& Rao, R.R. 1977. A Hand Book of Field and Herbarium Technique. Today \& Tomorrow's Publication, New Delhi.

Jain, S. \& Hajra, P. 1975. On the botany of Manas Wildlife Sanctuary in Assam. Bull. Bot. Surv. India 17: 75 - 86.

Kanjilal, U.N.; Kanjilal, P.C.; Das, A. \& De, R.N. 1934 - 1940. Flora of Assam. Vols. I-V. Govt Press, Shillong, India

Kar, A.; Borah, R.; Goswami, N.K \& Saharia, D. 2015. Inventorization of vascular plant diversity in Amchang Wildlife Sanctuary, Kamrup Metro district, Assam. Indian J. Appl. Res. 5(2): $48-60$.

Konwar, P.; Kakati, S.M. \& Saikia, P.K. 2009. Abundance of food plant species and food habits of Rhinoceros unicornis Linn. in Pobitora Wildlife Sanctuary, Assam, India. $J$. Threat. Taxa 1(9): $457-460$. 
Nath, S.K. 2012. Aquatic macrophytes of Laokhowa Wildlife Sanctuary, Assam, India. Intn. J. Eng. Res. App. 2: $1911-1913$.

Noss, R. 1992. The Wild lands Project: land conservation strategy. Wild Earth, Special Issue: $10-25$.

Sarkar, M. \& Devi, A. 2015. Assessment of plant diversity in Hollongapar Gibbon Wildlife Sanctuary, Assam, Northeast India. Indian For. 141(6): 648 - 659.

Sheikh, Y. 2018. Importance of National Park and Wildlife Sanctuary. Tathastu, April, Pp.1 -2 .

Shukla, R. 2016. The importance of protected areas. Frontline 33: 1 - 2.

Talukdar, S.R. \& Deori, C. 2017. Floristic Diversity of Laokhowa Wildlife Sanctuary, Assam, India. Nelumbo 59(2): $168-177$.

Tropicos. 2017. Tropicos. http://www.tropicos.org/Home.aspx. Acces-sed on: 2018-12-11. www.theplantlist.org [The Plant List. Version 1.1]

\section{APPENDIX - I}

\section{Angiospermic Flora of Borail Wildlife Sanctuary}

[Abbreviations used: Habit: $\mathrm{H}=\mathrm{Herb} ; \mathrm{S}=\mathrm{Shrub} ; \mathrm{Cl}=\mathrm{Climber} ; \mathrm{Cr}=$ Creeper; $\mathrm{L}=$ Lianas; $\mathrm{T}=$ Tree; $\mathrm{PA}=$ Palm; $\mathrm{G}=$ Grass; $\mathrm{SE}=$ Sedge; $\mathrm{B}=\mathrm{Bamboo}$; $\mathrm{EP}=$ Epiphytic. Habitat: $\mathrm{RS}=$ Road sides; SS: Stream side; HS= hill slopes; $\mathrm{RB}=$ River bank; $\mathrm{F}=$ Forest $]$

\begin{tabular}{|c|c|c|c|c|}
\hline $\begin{array}{l}\text { Sl. } \\
\text { no. }\end{array}$ & Name of the plants & Habit & Habitat & Distribution \\
\hline & \multicolumn{4}{|c|}{ DICOTYLEDONS } \\
\hline & ACANTHACEAE & & & \\
\hline 1 & Andrographis paniculata (Burm.f.) Wall. ex Nees & $\mathrm{H}$ & $\mathrm{F}$ & Common \\
\hline \multirow[t]{2}{*}{2} & Thunbergia grandiflora (Roxb. ex Rottl.) Roxb. & $\mathrm{L}$ & $\mathrm{F}$ & Very common \\
\hline & ACTINIDIACEAE & & & \\
\hline 3 & Saurauia armata Kurz & $\mathrm{T}$ & $\mathrm{F}$ & Rare \\
\hline \multirow[t]{2}{*}{4} & Saurauia roxburghii Wall. [Photo: 10$]$ & $\mathrm{T}$ & $\mathrm{F}$ & Rare \\
\hline & AMARANTHACEAE & & & \\
\hline 5 & Achyranthes aspera $\mathrm{L}$. & $\mathrm{H}$ & $\mathrm{RS}$ & Common \\
\hline 6 & Alternanthera sessilis (L.) R Br. ex DC. & $\mathrm{H}$ & $\mathrm{RS}$ & Common \\
\hline 7 & Amaranthus spinosus L. & $\mathrm{H}$ & $\mathrm{RS}$ & Common \\
\hline 8 & Amaranthus viridis $\mathrm{L}$. & $\mathrm{H}$ & RS & Common \\
\hline \multirow[t]{2}{*}{9} & Deeringia amaranthoides (Lam.) Merr. & $\mathrm{S}$ & HS & Rare \\
\hline & ANACARDIACEAE & & & \\
\hline 10 & Mangifera indica $\mathrm{L}$. & $\mathrm{T}$ & $\mathrm{F}$ & Common \\
\hline 11 & Spondias pinnata (L.f.) Kurz & $\mathrm{T}$ & $\mathrm{F}$ & Rare \\
\hline \multirow[t]{2}{*}{12} & Rhus chinensis Mill. & $\mathrm{T}$ & $\mathrm{F}$ & Rare \\
\hline & APOCYNACEAE & & & \\
\hline 13 & Alstonia scholaris (L.) R.Br. & $\mathrm{T}$ & $\mathrm{F}$ & Common \\
\hline 14 & Hoya verticillata (Vahl) G.Don & $\mathrm{Cr}$ & $\mathrm{F}$ & Common \\
\hline
\end{tabular}


A. Kar et al. 201

\begin{tabular}{|c|c|c|c|c|}
\hline $\begin{array}{l}\text { Sl. } \\
\text { no. }\end{array}$ & Name of the plants & Habit & Habitat & Distribution \\
\hline & \multicolumn{4}{|c|}{ DICOTYLEDONS (contd.) } \\
\hline 15 & $\begin{array}{l}\text { Tabernaemontana divaricata (L.) } \\
\text { R.Br. ex Roem. \& Schult. }\end{array}$ & $\mathrm{S}$ & $\mathrm{F}$ & Common \\
\hline 16 & Wrightia arborea (Dennst.) Mabb. & $\mathrm{T}$ & $\mathrm{F}$ & Rare \\
\hline \multirow[t]{2}{*}{17} & Wrightia antidysenterica (L.) R.Br. & $\mathrm{T}$ & $\mathrm{F}$ & Rare \\
\hline & ARALIACEAE & & & \\
\hline 18 & Aralia armata (Wall.ex G.Don) Seem. & $\mathrm{S}$ & $\mathrm{F}$ & Rare \\
\hline 19 & Trevesia palmata (Roxb. ex Lindl.) Vis. & $\mathrm{T}$ & F & Rare \\
\hline \multirow[t]{2}{*}{20} & Schefflera venulosa (Wight \& Arn.) Harms & $\mathrm{Cl}$ & $\mathrm{F}$ & Common \\
\hline & ASTERACEAE & & & \\
\hline 21 & Acmella oleracea (L.) R K Jansen & $\mathrm{H}$ & RS & Common \\
\hline 22 & Ageratum conyzoides (L.) L. & $\mathrm{H}$ & $\mathrm{RS}$ & Common \\
\hline 23 & Blumea lacera (Burm.f.) DC. & $\mathrm{H}$ & $\mathrm{RS}$ & Common \\
\hline 24 & Bidens bipinnata $\mathrm{L}$. & $\mathrm{H}$ & $\mathrm{RS}$ & Common \\
\hline 25 & Centipeda minima (L.) A.Braun \& Asch. & $\mathrm{S}$ & $\mathrm{RS}$ & Common \\
\hline 26 & Chromolaena odorata (L.) R.M. King \& H. Rob. & $\mathrm{S}$ & RS & Common \\
\hline 27 & $\begin{array}{l}\text { Crassocephalum crepidioides } \\
\text { (Benth.) S.Moore }\end{array}$ & $\mathrm{H}$ & $\mathrm{F}$ & Common \\
\hline 28 & Cyanthillium cinereum (L.) H.Rob. & $\mathrm{H}$ & $\mathrm{RS}$ & Common \\
\hline 29 & Dichrocephala integrifolia (L.f.) Kuntze & $\mathrm{H}$ & $\mathrm{RS}$ & Rare \\
\hline 30 & Erigeron canadensis $\mathrm{L}$. & $\mathrm{H}$ & $\mathrm{RS}$ & Rare \\
\hline 31 & Eclipta prostrata (L.) L. & $\mathrm{H}$ & RS & Common \\
\hline 32 & Elephantopus scaber L. & $\mathrm{H}$ & $\mathrm{F}$ & Common \\
\hline 33 & Helichrysum luteoalbum (L.) Rchb. & $\mathrm{H}$ & $\mathrm{RS}$ & Common \\
\hline 34 & Mikania micrantha Kunth. & $\mathrm{Cl}$ & $\mathrm{RS}$ & Common \\
\hline 35 & Sonchus wightianus DC. & $\mathrm{H}$ & RS & Common \\
\hline 36 & Sigesbeckia orientalis L. & $\mathrm{H}$ & $\mathrm{F}$ & Common \\
\hline 37 & Tithonia diversifolia (Hemsl.) A.Gray & $\mathrm{S}$ & $\mathrm{F}$ & Common \\
\hline 38 & Tridax procumbens $(\mathrm{L}.) \mathrm{L}$. & $\mathrm{H}$ & RS & Common \\
\hline \multirow[t]{2}{*}{39} & Xanthium strumarium L. & $\mathrm{H}$ & RS & Common \\
\hline & BALSAMINACEAE & & & \\
\hline \multirow[t]{2}{*}{40} & Impatiens balsamina $\mathrm{L}$. & $\mathrm{H}$ & RS & Rare \\
\hline & BEGONIACEAE & & & \\
\hline 41 & Begonia palmata D. Don & $\mathrm{H}$ & $\mathrm{F}$ & Rare \\
\hline 42 & Begonia picta $\mathrm{Sm}$. & $\mathrm{H}$ & $\mathrm{F}$ & Rare \\
\hline \multirow[t]{2}{*}{43} & Begonia roxburghii A.DC. & $\mathrm{H}$ & $\mathrm{F}$ & Rare \\
\hline & BIGNONIACEAE & & & \\
\hline 44 & Pajanelia longifolia (Willd.) K. Schum & $\mathrm{T}$ & $\mathrm{F}$ & Rare \\
\hline 45 & Oroxylum indicum (L.) Benth. ex Kurz & $\mathrm{T}$ & F & Rare \\
\hline \multirow[t]{2}{*}{46} & $\begin{array}{l}\text { Heterophragma adenophyllum (Wall ex G. Don) Seem ex } \\
\text { Benth. \& Hook.f. }\end{array}$ & $\mathrm{T}$ & $\mathrm{F}$ & Very rare \\
\hline & BURSERACEAE & & & \\
\hline 47 & Protium serratum (Wall. ex Colebr.) Engl. & $\mathrm{T}$ & F & Rare \\
\hline \multirow[t]{2}{*}{48} & Canarium strictum Roxb. & $\mathrm{T}$ & $\mathrm{F}$ & Rare \\
\hline & LEGUMINOSAE : CAESALPINIOIDEAE & & & \\
\hline 49 & Bauhinia vahlii Wight \& Arn. [Photo -14] & $\mathrm{L}$ & HS & Common \\
\hline 50 & Bauhinia purpurea $\mathrm{L}$ & $\mathrm{T}$ & HS & Common \\
\hline 51 & Bauhinia variegata $\mathrm{L}$. & $\mathrm{T}$ & $\mathrm{F}$ & Very Rare \\
\hline
\end{tabular}


202 Angiospermic flora of Barail Wildlife Sanctuar

\begin{tabular}{|c|c|c|c|c|}
\hline $\begin{array}{l}\text { Sl. } \\
\text { no. }\end{array}$ & Name of the plants & Habit & Habitat & Distribution \\
\hline 52 & Bauhinia scandens L.[Photo -7] & $\mathrm{L}$ & F & Very Rare \\
\hline 53 & Senna alata (L.) Roxb. & $\mathrm{S}$ & RS & Common \\
\hline 54 & Senna occidentalis (L.) Link & $\mathrm{H}$ & $\mathrm{RS}$ & Common \\
\hline 55 & Senna tora (L.) Roxb. & $\mathrm{H}$ & $\mathrm{RS}$ & Very Common \\
\hline 56 & Senna sophera (L.) Roxb. & $\mathrm{H}$ & $\mathrm{RS}$ & Common \\
\hline 57 & Cassia fistula $\mathrm{L}$ & $\mathrm{T}$ & RS & Rare \\
\hline \multirow[t]{2}{*}{58} & Cassia javanica $\mathrm{L}$. & $\mathrm{T}$ & RS & Rare \\
\hline & CALOPHYLLACEAE & & & \\
\hline \multirow[t]{2}{*}{59} & Mesua ferrea $\mathrm{L}$ & $\mathrm{T}$ & $\mathrm{F}$ & Common \\
\hline & CARYOPHYLLACEAEA & & & \\
\hline \multirow[t]{2}{*}{60} & Stellaria media (L.) Vill. & $\mathrm{H}$ & $\mathrm{F}$ & Very Common \\
\hline & CLEOMACEAE & & & \\
\hline 61 & Cleome gynandra $\mathrm{L}$. & $\mathrm{H}$ & $\mathrm{RS}$ & Common \\
\hline 62 & Crateva religiosa G.Forst. & $\mathrm{T}$ & $\mathrm{RB}$ & Rare \\
\hline \multirow[t]{2}{*}{63} & Cleome viscosa $\mathrm{L}$. & $\mathrm{H}$ & $\mathrm{RS}$ & Common \\
\hline & CONVOLVULACEAE & & & \\
\hline 64 & Argyreia nervosa (Burm.f.) Bojer & $\mathrm{Cl}$ & $\mathrm{F}$ & Very Common \\
\hline 65 & Cuscuta reflexa Roxb. & $\mathrm{Cl}$ & F & Very Common \\
\hline 66 & Ipomoea cheirophylla O'Donell & $\mathrm{Cl}$ & $\mathrm{F}$ & Very Common \\
\hline 67 & Ipomoea hederifolia $\mathrm{L}$. & $\mathrm{Cl}$ & F & Very Common \\
\hline 68 & Ipomoea cairica (L.) Sweet & $\mathrm{Cl}$ & F & Very Common \\
\hline 69 & Ipomoea purpurea (L.) Roth & $\mathrm{Cl}$ & F & Very Common \\
\hline 70 & Ipomoea nil (L.) Roth & $\mathrm{Cl}$ & F & Very Common \\
\hline \multirow[t]{2}{*}{71} & Poranopsis paniculata (Roxb.) Roberty & $\mathrm{Cl}$ & F & Very Common \\
\hline & CUCURBITACEAE & & & \\
\hline 72 & Coccinia grandis (L.) Voigt. & $\mathrm{Cl}$ & $\mathrm{F}$ & Rare \\
\hline 73 & Momordica charantia L. [Photo - 21] & $\mathrm{Cl}$ & F & Rare \\
\hline 74 & Trichosanthes cochinchinensis M.Roem. & $\mathrm{Cl}$ & $\mathrm{F}$ & Rare \\
\hline \multirow[t]{2}{*}{75} & Trichosanthes tricuspidata Lour. [Photo - 12] & $\mathrm{Cl}$ & $\mathrm{F}$ & Rare \\
\hline & DIPTEROCARPACEAE & & & \\
\hline \multirow[t]{2}{*}{76} & Dipterocarpus turbinatus C.F. Gaertn & $\mathrm{T}$ & $\mathrm{F}$ & Rare \\
\hline & EUPHORBIACEAE & & & \\
\hline 77 & Acalypha indica $\mathrm{L}$. & $\mathrm{H}$ & $\mathrm{RS}$ & Rare \\
\hline 78 & Croton bonplandianus Baill. & $\mathrm{H}$ & RS & Common \\
\hline 79 & Croton caudatus Geiseler & $\mathrm{S}$ & HS & Common \\
\hline 80 & Euphorbia hirta $\mathrm{L}$. & $\mathrm{H}$ & $\mathrm{RS}$ & Common \\
\hline 81 & Mallotus philippensis (Lam.) Müll.Arg. & $\mathrm{T}$ & $\mathrm{F}$ & Rare \\
\hline 82 & Mallotus tetracoccus (Roxb.) Kurz & $\mathrm{T}$ & $\mathrm{F}$ & Rare \\
\hline 83 & Mallotus nepalensis Müll.Arg. & & & \\
\hline 84 & Macaranga denticulata (Blume) Müll.Arg. & $\mathrm{T}$ & $\mathrm{F}$ & Common \\
\hline 85 & Macaranga peltata (Roxb.) Müll.Arg. & $\mathrm{T}$ & $\mathrm{F}$ & Common \\
\hline 86 & Macaranga indica Wight & $\mathrm{T}$ & $\mathrm{F}$ & Common \\
\hline \multirow[t]{2}{*}{87} & Mallotus nudiflorus (L.) Kulju \& Welzen & $\mathrm{T}$ & $\mathrm{F}$ & Common \\
\hline & LEGUMINOSAE : FABOIDEAE & & & \\
\hline 88 & Aeschynomene aspera $\mathrm{L}$. & $\mathrm{S}$ & RB & Rare \\
\hline 89 & Aeschynomene indica $\mathrm{L}$. & $\mathrm{H}$ & SS & Rare \\
\hline 90 & Alysicarpus vaginalis (L.) DC. & $\mathrm{H}$ & $\mathrm{RS}$ & Common \\
\hline 91 & Butea monosperma (Lam.) Taub. & $\mathrm{T}$ & $\mathrm{F}$ & Common \\
\hline
\end{tabular}


A. Kar et al. 203

\begin{tabular}{|c|c|c|c|c|}
\hline $\begin{array}{l}\text { Sl. } \\
\text { no. }\end{array}$ & Name of the plants & Habit & Habitat & Distribution \\
\hline 92 & Centrosema pubescens Benth. & $\mathrm{Cl}$ & $\mathrm{RS}$ & Rare \\
\hline 93 & Crotolaria pallida Aiton & $\mathrm{S}$ & $\mathrm{RS}$ & Common \\
\hline 94 & Desmodium gangeticum (L.) DC. & $\mathrm{S}$ & $\mathrm{RS}$ & Common \\
\hline 95 & Desmodium heterocarpon (L.) DC. & $\mathrm{S}$ & $\mathrm{RS}$ & Common \\
\hline 96 & Desmodium heterophyllum (Willd.) DC. & $\mathrm{H}$ & RS & Common \\
\hline 97 & $\begin{array}{l}\text { Hylodesmum podocarpum subsp. oxyphyllum (DC.) } \\
\text { H.Ohashi \& R.R.Mill }\end{array}$ & $\mathrm{S}$ & HS & Common \\
\hline 98 & Desmodium triflorum (L.) DC. & $\mathrm{H}$ & HS & Common \\
\hline 99 & Derris pachycarpa Merr. & $\mathrm{S}$ & HS & Rare \\
\hline 100 & Erythrina stricta Roxb. & $\mathrm{T}$ & RS & Common \\
\hline 101 & Mucuna pruriens (L.) DC. & $\mathrm{L}$ & $\mathrm{F}$ & Common \\
\hline 102 & Indigofera tinctoria $\mathrm{L}$. & $\mathrm{S}$ & HS & Rare \\
\hline 103 & Indigofera zollingeriana Miq. [Photo -6 ] & $\mathrm{T}$ & $\mathrm{F}$ & Rare \\
\hline 104 & Mucuna monosperma Roxb. ex Wight & $\mathrm{Cl}$ & F & Rare \\
\hline \multirow[t]{2}{*}{105} & Caesalpinia bonduc (L.) Roxb. & $\mathrm{S}$ & HS & Common \\
\hline & FAGACEAE & & & \\
\hline 106 & Castanopsis indica (Roxb. ex Lindl.) A.DC. & $\mathrm{T}$ & $\mathrm{F}$ & Rare \\
\hline 107 & Castanopsis lanceifolia (Oerst.) Hickel \& A.Camus & $\mathrm{T}$ & F & Rare \\
\hline \multirow[t]{2}{*}{108} & Quercus semiserrata Roxb. & $\mathrm{T}$ & $\mathrm{F}$ & Rare \\
\hline & GESNERIACEAE & & & \\
\hline \multirow[t]{2}{*}{109} & $\begin{array}{l}\text { Rhynchotechum ellipticum (Wall. ex D. Dietr.) A.DC. } \\
\text { [Photo - 18] }\end{array}$ & $\mathrm{S}$ & $\mathrm{F}$ & Very Common \\
\hline & LAMIACEAE & & & \\
\hline 110 & Anisomeles indica (L.) Kuntze & $\mathrm{H}$ & $\mathrm{F}$ & Common \\
\hline 111 & Callicarpa tomentosa $(\mathrm{L}.) \mathrm{L}$. & $\mathrm{T}$ & $\mathrm{F}$ & Common \\
\hline 112 & Clerodendrum glandulosum Lindl. & $\mathrm{S}$ & $\mathrm{F}$ & Common \\
\hline 113 & Clerodendrum hastatum (Roxb.) Lindl. & $\mathrm{S}$ & $\mathrm{F}$ & Rare \\
\hline 114 & Clerodendrum indicum (L.) Kuntze & $\mathrm{S}$ & $\mathrm{F}$ & Common \\
\hline 115 & Clerodendrum infortunatum $\mathrm{L}$. & $\mathrm{H}$ & $\mathrm{RS}$ & Very Common \\
\hline 116 & Gmelina arborea Roxb. & $\mathrm{T}$ & $\mathrm{F}$ & Rare \\
\hline 117 & Holmskioldia sanguinea Retz. & $\mathrm{S}$ & $\mathrm{F}$ & Common \\
\hline 118 & Rotheca farinosa (Roxb.) Govaerts & $\mathrm{T}$ & $\mathrm{F}$ & Rare \\
\hline 119 & Rotheca serrata (L.) Steane \& Mabb. & $\mathrm{S}$ & HS & Common \\
\hline \multirow[t]{2}{*}{120} & Tectona grandis L.f. & $\mathrm{T}$ & $\mathrm{F}$ & Common \\
\hline & LAURACEAE & & & \\
\hline 121 & Actinodaphne obovata (Nees) Blume & $\mathrm{T}$ & $\mathrm{F}$ & Rare \\
\hline 122 & Cinnamomum bejolghota (Buch.-Ham.) Sweet & $\mathrm{T}$ & $\mathrm{F}$ & Rare \\
\hline \multirow[t]{2}{*}{123} & Litsea glutinosa (Lour.) C.B. Rob. & $\mathrm{T}$ & $\mathrm{F}$ & Rare \\
\hline & LECYTHIDACEAE & & & \\
\hline \multirow[t]{2}{*}{124} & Careya arborea Roxb. & $\mathrm{T}$ & $\mathrm{F}$ & Rare \\
\hline & LORANTHACEAE & & & \\
\hline \multirow[t]{2}{*}{125} & Dendrophthoe falcata (L.f.) Ettingsh. & $\mathrm{H}$ & $\mathrm{P}$ & Rare \\
\hline & LYTHRACEAE & & & \\
\hline 126 & Duabanga grandiflora (Roxb. ex DC.) Walp. & $\mathrm{T}$ & $\mathrm{F}$ & Common \\
\hline 127 & Lagerstroemia parviflora Roxb. & $\mathrm{T}$ & $\mathrm{F}$ & Rare \\
\hline \multirow[t]{2}{*}{128} & Lagerstroemia speciosa (L.) Pers. & $\mathrm{T}$ & $\mathrm{F}$ & Rare \\
\hline & MALVACEAE & & & \\
\hline 129 & Abelmoschus moschatus Medik. & $\mathrm{H}$ & $\mathrm{F}$ & Common \\
\hline
\end{tabular}


204 Angiospermic flora of Barail Wildlife Sanctuar

\begin{tabular}{|c|c|c|c|c|}
\hline $\begin{array}{l}\text { Sl. } \\
\text { no. }\end{array}$ & Name of the plants & Habit & Habitat & Distribution \\
\hline 130 & Abroma augusta (L..) L.f. [Photo - 15] & $\mathrm{S}$ & $\mathrm{F}$ & Rare \\
\hline 131 & Abutilon indicum (Link) Sweet & $\mathrm{H}$ & $\mathrm{RS}$ & Common \\
\hline 132 & Firmiana simplex (L.) W.Wight & $\mathrm{T}$ & $\mathrm{F}$ & Common \\
\hline 133 & Sida acuta Burm.f & $\mathrm{H}$ & $\mathrm{RS}$ & Common \\
\hline 134 & Sida cordifolia $\mathrm{L} .$. & $\mathrm{H}$ & RS & Rare \\
\hline 135 & Sida rhombifolia $\mathrm{L}$. & $\mathrm{H}$ & $\mathrm{RS}$ & Rare \\
\hline 136 & Sterculia villosa Roxb. & $\mathrm{T}$ & $\mathrm{F}$ & Common \\
\hline \multirow[t]{2}{*}{137} & Urena lobata $\mathrm{L}$. & $\mathrm{H}$ & $\mathrm{RS}$ & Common \\
\hline & MELASTOMATACEAE & & & \\
\hline 138 & Medinilla assamica (C.B. Clarke) C. Chen [Photo - 11] & $\mathrm{S}$ & F & Rare \\
\hline 139 & Melastoma malabathricum $\mathrm{L}$. & $\mathrm{S}$ & RS & Common \\
\hline 140 & Osbeckia nepalensis Hook. & $\mathrm{S}$ & HS & Rare \\
\hline \multirow[t]{2}{*}{141} & Osbeckia stellata Buch.-Ham. ex D.Don & & & \\
\hline & MELIACEAE & & & \\
\hline 142 & Aphanamixis polystachya (Wall.) R. Parker & $\mathrm{T}$ & HS & Rare \\
\hline 143 & Dysoxylum gotadhora (Buch.-Ham.) Mabb. & $\mathrm{T}$ & HS & Rare \\
\hline 144 & Melia azedarach $\mathrm{L}$ & $\mathrm{T}$ & HS & Common \\
\hline \multirow[t]{2}{*}{145} & Toona ciliata M Roem. & $\mathrm{T}$ & $\mathrm{F}$ & Common \\
\hline & MENISPERMACEAE & & & \\
\hline \multirow[t]{2}{*}{146} & Tinospora crispa (L.) Hook.f. \& Thomson & $\mathrm{L}$ & $\bar{F}$ & Common \\
\hline & LEGUMINOSAE : MIMOSAOIDEAE & & & \\
\hline 147 & Acacia concinna DC. & $\mathrm{T}$ & $\mathrm{F}$ & Common \\
\hline 148 & Vachellia farnesiana (L.) Wight \& Arn. & $\mathrm{T}$ & $\mathrm{F}$ & Common \\
\hline 149 & Senegalia pennata (L.) Maslin & $\mathrm{T}$ & $\mathrm{F}$ & Common \\
\hline 150 & Albizia amara (Roxb.) Boivin & $\mathrm{T}$ & $\mathrm{F}$ & Common \\
\hline 151 & Albizia lebbek (L.) Benth. & $\mathrm{T}$ & $\mathrm{F}$ & Common \\
\hline 152 & Albizia lucidor (Steud.) I.C. Nielsen & $\mathrm{T}$ & $\mathrm{F}$ & Common \\
\hline 153 & Albizia odoratissima (L.f.)Benth. & $\mathrm{T}$ & $\mathrm{F}$ & Common \\
\hline 154 & Albizia procera (Roxb.) Benth. & $\mathrm{T}$ & $\mathrm{F}$ & Common \\
\hline 155 & Albizia saman (Jacq.) Merr. & $\mathrm{T}$ & $\mathrm{F}$ & Common \\
\hline 156 & Mimosa diplotricha Sauvalle & $\mathrm{S}$ & $\mathrm{RS}$ & Common \\
\hline \multirow[t]{2}{*}{157} & Mimosa pudica $\mathrm{L}$ & $\mathrm{H}$ & RS & Common \\
\hline & MORACEAE & & & \\
\hline 158 & Artocarpus chama Buch.-Ham. & $\mathrm{T}$ & $\mathrm{F}$ & Rare \\
\hline 159 & Artocarpus lacucha Roxb. ex Buch.-Ham. & $\mathrm{T}$ & F & Rare \\
\hline 160 & Ficus auriculata Lour. & $\mathrm{T}$ & $\mathrm{F}$ & Common \\
\hline 161 & Ficus benjamina L. & $\mathrm{T}$ & $\mathrm{F}$ & Rare \\
\hline 162 & Ficus hispida L.f. & $\mathrm{T}$ & $\mathrm{F}$ & Common \\
\hline 163 & Ficus hirta Vahl & $\mathrm{T}$ & F & Rare \\
\hline 164 & Ficus racemosa $\mathrm{L}$. & $\mathrm{T}$ & $\mathrm{F}$ & Common \\
\hline 165 & Ficus religiosa $\mathrm{L}$. & $\mathrm{T}$ & $\mathrm{F}$ & Common \\
\hline 166 & Ficus benghalensis L. & $\mathrm{T}$ & $\mathrm{F}$ & Common \\
\hline 167 & Ficus elastica Roxb. ex Hornem. & $\mathrm{T}$ & F & Common \\
\hline \multirow[t]{2}{*}{168} & Ficus semicordata Buch.-Ham. ex Sm. & $\mathrm{T}$ & $\mathrm{F}$ & Common \\
\hline & MYRTACEAE & & & \\
\hline \multirow[t]{2}{*}{169} & Syzygium cumini (L.) Skeels & $\mathrm{T}$ & $\mathrm{F}$ & Common \\
\hline & OLACACEAE & & & \\
\hline 170 & Olax acuminata Wall. ex Benth. & $\mathrm{S}$ & $\mathrm{F}$ & Rare \\
\hline
\end{tabular}


A. Kar et al. 205

\begin{tabular}{|c|c|c|c|c|}
\hline Sl. & Name of the plants & Habit & Habitat & Distribution \\
\hline & OLEACEAE & & & \\
\hline \multirow[t]{2}{*}{171} & Jasminum multiflorum (Burm.f.) Andrews [Photo -17] & $\mathrm{Cl}$ & $\mathrm{F}$ & Rare \\
\hline & ONAGRACEAE & & & \\
\hline 172 & Ludwigia hyssopifolia (G.Don) Exell & $\mathrm{H}$ & SS & Common \\
\hline 173 & Ludwigia octovalvis (Jacq.) P.H.Raven & $\mathrm{H}$ & SS & Rare \\
\hline \multirow[t]{2}{*}{174} & Ludwigia prostrata $\mathrm{Roxb}$ & $\mathrm{H}$ & SS & Common \\
\hline & PENTAPHYLLACEAE & & & \\
\hline \multirow[t]{2}{*}{175} & Eurya acuminata DC. & $\mathrm{S}$ & $\mathrm{F}$ & Rare \\
\hline & PHYLLANTHACEAE & & & \\
\hline 176 & Antidesma acidum Retz. & $\mathrm{S}$ & $\mathrm{F}$ & Rare \\
\hline 177 & Bridelia retusa (L.) A. Juss. & $\mathrm{T}$ & HS & Rare \\
\hline 178 & Phyllanthus amarus Schumach. \& Thonn. & $\mathrm{H}$ & RS & Common \\
\hline \multirow[t]{2}{*}{179} & Phyllanthus emblica $\mathrm{L}$. & $\mathrm{T}$ & $\mathrm{F}$ & Rare \\
\hline & PIPERACEAE & & & \\
\hline 180 & Peperomia pellucida (L.) Kunth & $\mathrm{H}$ & RS & Common \\
\hline 181 & Piper acutistigmum C.DC. [Photo -5] & $\mathrm{Cl}$ & $\mathrm{F}$ & Common \\
\hline \multirow[t]{2}{*}{182} & Piper thomsonii (C.DC.) Hook.f. & $\mathrm{Cl}$ & $\mathrm{F}$ & Rare \\
\hline & POLYGONACEAE & & & \\
\hline 183 & Persicaria barbata (L.) H.Hara & $\mathrm{H}$ & $\mathrm{F}$ & Common \\
\hline 184 & Persicaria chinensis (L.) H.Gross & $\mathrm{Cl}$ & $\mathrm{F}$ & Common \\
\hline 185 & Persicaria hydropiper (L.) Dilarbre & $\mathrm{H}$ & $\mathrm{SS}$ & Common \\
\hline 186 & Persicaria strigosa (R.Br.) Nakai & $\mathrm{H}$ & $\mathrm{F}$ & Common \\
\hline 187 & Polygonum perfoliatum L. & $\mathrm{Cl}$ & $\mathrm{F}$ & Rare \\
\hline 188 & Polygonum plebeium $\mathrm{R} . \mathrm{Br}$. & $\mathrm{H}$ & $\mathrm{F}$ & Common \\
\hline \multirow[t]{2}{*}{189} & Rumex maritimus L. & $\mathrm{H}$ & $\mathrm{RS}$ & Common \\
\hline & RHAMNACEAE & & & \\
\hline \multirow[t]{2}{*}{190} & Ziziphus jujuba Mill. & $\mathrm{T}$ & $\mathrm{F}$ & Common \\
\hline & RUBIACEAE & & & \\
\hline 191 & Cephalanthus occidentalis L. & $\mathrm{T}$ & $\mathrm{F}$ & Rare \\
\hline 192 & Dentella repens (L.) J.R.Forst. \& G.Forst. & $\mathrm{H}$ & RS & Common \\
\hline 193 & Haldina cordifolia (Roxb.) Ridsdale & $\mathrm{T}$ & $\mathrm{F}$ & Rare \\
\hline 194 & Mussaenda glabra Vahl & $\mathrm{S}$ & $\mathrm{F}$ & Common \\
\hline 195 & Oldenlandia diffusa (Willd.) Roxb. & $\mathrm{H}$ & $\mathrm{RS}$ & Common \\
\hline 196 & Mussaenda macrophylla Wall. & $\mathrm{S}$ & $\mathrm{F}$ & Rare \\
\hline 197 & Mussaenda roxburghii Hook.f. & $\mathrm{S}$ & $\mathrm{F}$ & Common \\
\hline 198 & Paederia foetida $\mathrm{L}$. & $\mathrm{Cl}$ & $\mathrm{F}$ & Common \\
\hline 199 & Paederia scandens (Lour.) Merr. & $\mathrm{Cl}$ & $\mathrm{F}$ & Common \\
\hline \multirow[t]{2}{*}{200} & Spermacoce hispida L. & $\mathrm{H}$ & $\mathrm{RS}$ & Common \\
\hline & RUTACEAE & & & \\
\hline 201 & Zanthoxylum acanthopodium DC. & $\mathrm{T}$ & $\mathrm{F}$ & Rare \\
\hline 202 & Zanthoxylum oxyphyllum Edgew. & $\mathrm{T}$ & $\mathrm{F}$ & Rare \\
\hline \multirow[t]{2}{*}{203} & Zanthoxylum rhetsa (Roxb.) DC. & $\mathrm{T}$ & $\mathrm{F}$ & Rare \\
\hline & SAPINDACEAE & & & \\
\hline \multirow[t]{2}{*}{204} & Xerospermum noronhianum Blume & $\mathrm{T}$ & $\mathrm{F}$ & Common \\
\hline & SOLANACEAE & & & \\
\hline 205 & Nicotiana plumbaginifolia Viv. & $\mathrm{H}$ & $\mathrm{RS}$ & Rare \\
\hline 206 & Physalis minima L. & $\mathrm{H}$ & $\mathrm{RS}$ & Rare \\
\hline 207 & Solanum aculeatissimum Jacq. & $\mathrm{H}$ & RS & Common \\
\hline
\end{tabular}


206 Angiospermic flora of Barail Wildlife Sanctuar

\begin{tabular}{|c|c|c|c|c|}
\hline $\begin{array}{l}\text { Sl. } \\
\text { no. }\end{array}$ & Name of the plants & Habit & Habitat & Distribution \\
\hline 208 & Solanum americanum Mill. & $\mathrm{H}$ & $\mathrm{RS}$ & Common \\
\hline 209 & Solanum indicum $\mathrm{L}$. & $\mathrm{H}$ & HS & Common \\
\hline \multirow[t]{2}{*}{210} & Solanum torvum $\mathrm{Sw}$. & $\mathrm{S}$ & HS & Common \\
\hline & THEACEAE & & & \\
\hline 211 & Camellia kissi Wall. & $\mathrm{S}$ & $\mathrm{F}$ & Rare \\
\hline \multirow[t]{2}{*}{212} & Schima wallichii Choisy & $\mathrm{T}$ & HS & Rare \\
\hline & VERBENACEAE & & & \\
\hline 213 & Lantana camara $\mathrm{L}$. & $\mathrm{S}$ & $\mathrm{RS}$ & Common \\
\hline 214 & Lippia alba (Mill.) N.E.Br. ex Britton \& P.Wilson & $\mathrm{S}$ & RS & Rare \\
\hline 215 & Phyla nodiflora (L.) Greene & $\mathrm{H}$ & RS & Rare \\
\hline \multirow[t]{2}{*}{216} & Stachytarpheta jamaicensis (L.) Vahl & $\mathrm{H}$ & $\mathrm{RS}$ & Common \\
\hline & VITACEAE & & & \\
\hline 217 & Cissus adnata Roxb. & $\mathrm{Cl}$ & $\mathrm{F}$ & Common \\
\hline 218 & Cayratia trifolia (L.) Domin & $\mathrm{Cl}$ & $\mathrm{F}$ & Very common \\
\hline 219 & Leea asiatica (L.) Ridsdale & $\mathrm{S}$ & $\mathrm{F}$ & Common \\
\hline 220 & Leea indica (Burm.f.) Merr. & $\mathrm{S}$ & F & Very common \\
\hline \multirow[t]{2}{*}{221} & Tetrastigma pedunculare (Wall. ex Lowson) Planch. & $\mathrm{Cl}$ & $\mathrm{F}$ & Rare \\
\hline & URTICACEAE & & & \\
\hline 222 & Boehmeria macrophylla Hornem. [Photo -13] & $\mathrm{S}$ & HS & Rare \\
\hline 223 & Debregeasia longifolia (Burm.f) Wedd. & $\mathrm{S}$ & HS & Rare \\
\hline 224 & Elatostema sessile J.R. Forst. \& G. Frost. & $\mathrm{H}$ & SS & Rare \\
\hline 225 & Pouzolzia hirta Blume ex Hassk & $\mathrm{H}$ & RS & Common \\
\hline 226 & Sarcochlamys pulcherrima Gaudich. [Photo -19] & $\mathrm{S}$ & HS & Common \\
\hline \multirow[t]{3}{*}{227} & Girardinia diversifolia (Link) Friis & $\mathrm{S}$ & $\mathrm{F}$ & Common \\
\hline & \multicolumn{4}{|c|}{ MONOCOTYLEDONS } \\
\hline & ARACEAE & & & \\
\hline 228 & Alocasia cucullata (Lour.) G.Don & $\mathrm{H}$ & HS & Rare \\
\hline 229 & Colocasia fallax Schott & $\mathrm{H}$ & $\mathrm{F}$ & Rare \\
\hline 230 & Homalomena aromatica (Spreng.) Schott [Photo -16] & $\mathrm{H}$ & HS & Rare \\
\hline 231 & Pothos scandens L. & $\mathrm{Cl}$ & $\mathrm{F}$ & Common \\
\hline 232 & Remusatia pumila (D.Don) H.Li \& A.Hay [Photo -4] & $\mathrm{H}$ & HS & Common \\
\hline 233 & Remusatia hookeriana Schott & $\mathrm{H}$ & HS & Rare \\
\hline \multirow[t]{2}{*}{234} & Rhaphidophora decursiva (Roxb.) Schott & $\mathrm{Cl}$ & $\mathrm{F}$ & Common \\
\hline & ARECACEAE & & & \\
\hline 235 & Arenga pinnata (Wurmb) Merr. [Photo -25] & PA & $\mathrm{F}$ & Rare \\
\hline 236 & Caryota urens $\mathrm{L}$. & PA & $\mathrm{F}$ & Common \\
\hline \multirow[t]{2}{*}{237} & Licuala spinosa Wurmb [Photo -22] & $\mathrm{PA}$ & $\mathrm{F}$ & Common \\
\hline & COSTACEAE & & & \\
\hline \multirow[t]{2}{*}{238} & $\begin{array}{l}\text { Cheilocostus speciosus (J.Koenig) C.D. Specht. [Photo - } \\
\text { 20] }\end{array}$ & $\mathrm{H}$ & $\mathrm{F}$ & Rare \\
\hline & CYPERACEAE & & & \\
\hline 239 & Carex dimorpholepis Steud. & SE & RS & Common \\
\hline 240 & Cyperus compactus Retz. & SE & $\mathrm{RS}$ & Common \\
\hline 241 & Cyperus compressus $\mathrm{L}$. & SE & RS & Common \\
\hline 242 & Cyperus cyperinus (Retz.) Suringar & $\mathrm{SE}$ & $\mathrm{RS}$ & Common \\
\hline 243 & Fimbristylis eragrostis (Nees) Hance & SE & RS & Common \\
\hline \multirow[t]{2}{*}{244} & Kyllinga brevifolia Rottb. & SE & $\mathrm{RS}$ & Common \\
\hline & DIOSCOREACEAE & & & \\
\hline
\end{tabular}


A. Kar et al. 207

\begin{tabular}{|c|c|c|c|c|}
\hline $\begin{array}{l}\text { Sl. } \\
\text { no. }\end{array}$ & Name of the plants & Habit & Habitat & Distribution \\
\hline 245 & Dioscorea alata L. & $\mathrm{Cl}$ & $\mathrm{F}$ & Common \\
\hline 246 & Dioscorea bulbifera L. & $\mathrm{Cl}$ & F & Common \\
\hline 247 & Dioscorea deltoidea Wall. ex Griseb. & $\mathrm{Cl}$ & $\mathrm{F}$ & Common \\
\hline 248 & Dioscorea esculenta (Lour.) Burkill & $\mathrm{Cl}$ & $\mathrm{F}$ & Common \\
\hline 249 & Dioscorea hamiltonii Hook.f. & $\mathrm{Cl}$ & $\mathrm{F}$ & Common \\
\hline \multirow[t]{2}{*}{250} & Dioscorea pentaphylla L. [Photo -3] & $\mathrm{Cl}$ & $\mathrm{F}$ & Rare \\
\hline & LILIACEAE & & & \\
\hline \multirow[t]{2}{*}{251} & Asparagus racemosus Willd. & $\mathrm{Cl}$ & $\mathrm{F}$ & Rare \\
\hline & MARANTACEAE & & & \\
\hline \multirow[t]{2}{*}{252} & Phrynium pubinerve Blume [Photo -9] & $\mathrm{H}$ & $\mathrm{F}$ & Rare \\
\hline & MUSACEAE & & & \\
\hline \multirow[t]{2}{*}{253} & Musa balbisiana Colla & $\mathrm{H}$ & $\mathrm{F}$ & Very common \\
\hline & ORCHIDACEAE & & & \\
\hline 254 & Arundina graminifolia (D.Don) Hochr. [Photo -8, 23] & $\mathrm{S}$ & HS & Common \\
\hline 255 & Dendrobium densiflorum Lindl. & $\mathrm{H}$ & EP & Rare \\
\hline 256 & Papilionanthe teres (Roxb.) Schltr. & $\mathrm{H}$ & EP & Common \\
\hline \multirow[t]{2}{*}{257} & Rhynchostylis retusa (L.) Blume & $\mathrm{H}$ & EP & Common \\
\hline & PANDANACEAE & & & \\
\hline \multirow[t]{2}{*}{258} & Pandanus odorifer (Forssk.) Kuntze [Photo -2] & $\mathrm{T}$ & $\mathrm{F}$ & Rare \\
\hline & POACEAE & & & \\
\hline 259 & Arundo donax $\mathrm{L}$. & G & SS & Common \\
\hline 260 & Bambusa balcooa Roxb. & $\mathrm{B}$ & $\mathrm{F}$ & Common \\
\hline 261 & Bambusa cacharensis R.B. Majumdar & $\mathrm{B}$ & $\mathrm{F}$ & Rare \\
\hline 262 & Bambusa tulda Roxb. & $\mathrm{B}$ & $\mathrm{F}$ & Common \\
\hline 263 & Cynodon dactylon (L.) Pers. & $\mathrm{G}$ & $\mathrm{RS}$ & Common \\
\hline 264 & Eleusine indica (L.) Gaertn & $\mathrm{G}$ & $\mathrm{RS}$ & Common \\
\hline 265 & Eragrostis pilosa (L.) P.Beauv & $\mathrm{G}$ & $\mathrm{SS}$ & Common \\
\hline 266 & Imperata cylindrica (L.) Raeusch. & $\mathrm{G}$ & SS & Common \\
\hline 267 & Melocanna baccifera (Roxb.) Kurz & $\mathrm{B}$ & $\mathrm{F}$ & Common \\
\hline 268 & Oplismenus compositus (L.) P.Beauv. & $\mathrm{G}$ & $\mathrm{RS}$ & Common \\
\hline 269 & Panicum brevifolium $\mathrm{L}$. & $\mathrm{G}$ & $\mathrm{RS}$ & Common \\
\hline 270 & Phragmites karka (Retz.) Trin. ex Steud & $\mathrm{G}$ & $\mathrm{RS}$ & Common \\
\hline 271 & Schizostachyum dullooa (Gamble) R.B.Majumdar & $\mathrm{B}$ & $\mathrm{F}$ & Common \\
\hline 272 & Setaria palmifolia (J.Koenig) Stapf & G & $\mathrm{RS}$ & Common \\
\hline \multirow[t]{2}{*}{273} & Thysanolaena latifolia (Roxb. ex Hornem.) Honda & $\mathrm{G}$ & $\mathrm{RS}$ & Common \\
\hline & SMILACACEAE & & & \\
\hline 274 & Smilax china $\mathrm{L}$. & $\mathrm{Cl}$ & $\mathrm{F}$ & Rare \\
\hline 275 & Smilax perfoliata Lour. & $\mathrm{Cl}$ & $\mathrm{F}$ & Rare \\
\hline \multirow[t]{2}{*}{276} & Smilax zeylanica $\mathrm{L}$. & $\mathrm{Cl}$ & $\mathrm{F}$ & Rare \\
\hline & STEMONACEAE & & & \\
\hline \multirow[t]{2}{*}{277} & Stemona tuberosa Lour. & $\mathrm{Cl}$ & $\mathrm{F}$ & Rare \\
\hline & ZINGIBERACEAE & & & \\
\hline 278 & Hedychium coccineum Buch-Ham ex Sm. & $\mathrm{H}$ & F & Rare \\
\hline 279 & Globba clarkei Baker & $\mathrm{H}$ & $\mathrm{F}$ & Rare \\
\hline 280 & Globba multiflora Wall. ex Baker & $\mathrm{H}$ & $\mathrm{F}$ & Rare \\
\hline 281 & Globba racemosa $\mathrm{Sm}$. & $\mathrm{H}$ & $\mathrm{F}$ & Rare \\
\hline
\end{tabular}

